\section{Performance of Piezoelectric Transducers in Terms of Amplitude and Waveform}

\section{Abstract}

For seismic physical modeling, mostly piezoelectric transducers (PETs) are used as sources and receivers. Their properties have significant effects on the data, especially if they are to be processed as seismic data: 1. Strong resonance at one frequency causes a ringy signal and a narrow frequency band. 2 . The pronounced directionality effectively limits the offsets at which energy arrives. 3. Because the dimension of the transducer with 12 $\mathrm{mm}$ is bigger than the wavelength $(1.5-10 \mathrm{~mm})$, the recorded waveform changes with offset. To reduce the pronounced directionality of the transducers at ultrasonic frequencies, we have designed PETs that have a smaller effective diameter than traditional ones. To test their applicability for laboratory seismic profiling, we test their frequency sensitivity, their directionality, and the change of waveform as a function of offset due to their size compared to the wavelengths. The experiments show that the PETs produce their best quality data at frequencies around 350-550 $\mathrm{kHz}$ and source-receiver offsets $\leq 14 \mathrm{~cm}$. For these frequencies, the amplitudes decay to ringing noise level at incidence angles of $<35^{\circ}$; for a $10 \mathrm{~cm}$ deep reflector that results in a $14 \mathrm{~cm}$ source-receiver offset. For these offsets and frequencies, the spacious dimension of the PETs does not cause the waveform to change such that further processing is compromised. Also, we present an analytical solution to the changing waveform problem which predicts the temporal divergence of the signal as an additional resolution limit to the Fresnel effect; the loss of high frequencies is not only caused by attenuation, but is also due to the spacious dimension of the sensors.

\subsection{Introduction}

Since the 1920s, seismic physical modeling has been a successful tool for research in wave phenomena (i.e. the kinematics of wave propagation and the validation of wave theoretical predictions). In the first experiments, optical methods were used to record surface motion (Tsuboi, 1994) or wavefronts through transparent media (Rieber, 1936, 1937; Schmidt, 1939).

These experiments were performed on models such as rods (1-D), or elastic plates (2-D and 3D) (e.g., Berryman et al., 1958; Redwood, 1960; Purnell, 1986; Zhang et al., 1996; Wandler et al., 2007, among many others). However, before 2001 virtually all models were made of solid materials, and thus were static. Dynamic models in which the material is deformed while monitoring require viscous or granular media such as sand, but severe attenuation and scattering of seismic waves in sand prevented the application of seismic imaging methods on sandbox models (Purnell, 1986). Sherlock (1999) and Sherlock and Evans (2001) were the first to try to overcome these problems and performed zerooffset seismic surveys at the mm-scale using piezoelectric transducers (PET) on sandbox models.

One reason to repeatedly try to perform seismic imaging on sandbox models is that these dynamic sandbox experiments have provided qualitative and quantitative insights into specific geological problems (e.g., Kovi, 1995, 1997; Storti et al., 2000; Lohrmann et al., 2003; 
Gartrell et al., 2005; Hoth et al., 2007). So far, the internal structure of sandbox models can only be directly observed in 2-D profiles along glass walls confining the 2-D models or indirectly by surface observations (e.g. PIV - particle image velocimetry; Adam et al., 2005) or by $\mathrm{X}$ ray tomography (Colletta et al., 1991). An improved seismic imaging system could provide non-invasive albeit less resolved 3-D information.

To achieve this objective, a new small-scale seismic apparatus for laboratory use was designed and developed, composed of a water tank, a PC control unit including PETs, and a positioning system (Krawczyk et al., 2007) with the ultimate goal to apply 3-D seismic and seismological imaging methods to sandbox models subject to deformation. These models are made of saturated granular materials so that deformation can take place. Resulting structures like shear bands are 2-3 $\mathrm{mm}$ wide and the layers have a thickness of a few millimeters to centimeters. Hence, the source frequencies need to be between $250 \mathrm{kHz}$ and $1 \mathrm{MHz}$ to generate waves with wavelengths between 6 to $1.5 \mathrm{~mm}$, so that they are able to resolve these structures. Higher frequencies generate wavelengths that are approximately as big as the grain size of the material, so that each grain scatters arriving energy causing high attenuation.

When doing seismic physical modeling of solid or granular models, three aspects require particular attention: Scaling, transducer and material properties. In contrast to the continuing discussion about scaling factors within the geological physical modeling community, scaling for seismic physical models is trivial: Length and time scale factors are arbitrary, as long as the ratio of geological feature size to wavelength is the same in both the field and the model (Ebrom and McDonald, 1994). In nature as well as in the model, only the Nyquist criterion must be obeyed for temporal and spatial sampling. Regarding the equipment, most experimenters used electromechanical transducers, beginning with the work of Kaufman and Roever (1994). Sometimes sparks (Kaufman and Roever, 1994; Hilterman, 1970) were used as seismic sources, but mostly PETs have served both as sources and receivers, such that the results are in the same form as field records (Riznichenko, 1994; O'Brien and Symes, 1994). However, the proper scaling of source and receiver dimension to wavelength is inevitably violated (in the field, individual sources and receivers are generally small compared to a wavelength), which imposes limitations on the use of PETs in seismic physical modeling:

- strong resonance at one frequency (i.e. restricted bandwidth),

- pronounced directionality, and

- source and receiver dimensions in the same order of magnitude as the wavelengths.

Furthermore, the material available for seismic physical modeling has several limiting effects: The parameter ranges for velocity and density are limited to those materials that are available or can be fabricated. Additionally, the attenuative properties and scattering effects of modeling materials cause substantial weakening of the received signals. Good source and receiver coupling to the model can be achieved by performing the experiments in a water tank.

The effects of the source and receiver dimension have been frequently neglected or dismissed in published physical modeling studies, despite the fact that they can have a first order effect on the data. Dellinger and Vernik (1994) numerically modeled whether experiments to measure velocities of layered rocks are more likely to measure the group velocity or the phase velocity of p- and s-waves. In their models they addressed the effect of a spatial source on wave propagation, and their Figure 5 shows nicely the waveform divergence with increasing offset or increasing source and receiver size at zero offset. However, they explained it solely as a result 
of the anisotropic wave propagation in a layered medium with a slope and did not stress, that part of this divergence occurs even in a homogeneous medium.

Within the scope of this publication, we test the properties of our PETs with respect to their use in seismic reflection surveys of models and geometries as described above. We analyze the above mentioned properties analytically and experimentally and discuss the limitations and opportunities imposed by them.

\subsection{Effects of piezoelectric transducers in ultrasonic experiments}

The three limitations of piezoelectric transducers (PET), namely the strong resonance at one frequency, large dimensions and directionality, have the following effects on the seismic signal:

1. ringy signal at resonance/eigen frequency,

2. changing waveform with offset (temporal divergence),

3. high attenuation with offset (spatial divergence) in the plane parallel to the emitter surface.

\subsubsection{Resonance frequency}

Due to the piezoelectric effect, a piezocrystal can be excited to oscillate by applying AC. The amplitude of the oscillation is dependent on the source frequency and has its maximum at the resonance frequency of the PET (Krautkrämer and Krautkrämer, 1986). In order to reduce the oscillation subsequent to the excitation period, particularly at resonance frequency, the PETs are damped by a tungsten/resin filling. However, some ringing remains. This can be either reduced by a deconvolution filter, or the signal can be recorded at all angles and then used as a cross-correlation wavelet.

\subsubsection{Changing waveform}

The shape of a wavelet propagating from a source to a receiver changes with offset when their dimensions are bigger or of the same size as the emitted wavelength, because the length difference of the rays may be well over a wavelength $\lambda$, as illustrated by the two sample rays $r_{1}$ and $r_{2}$ in Figure 2.1. Hence, we have to consider each point of the source as an individual source point and assume that it emits energy at the same time (or at least within one or two sampling intervals) as all other source points. Due to different raypath lengths to any of the receiver points, the energy emitted at one instant arrives over a continuous time period which is increasing with offset. We call this effect "temporal divergence" following the concept of "spherical divergence" where energy propagating from a source point is distributed over a greater area. This effect is very similar to the Fresnel zone effect, only that the Fresnel zone is defined as the area to within half a wavelength around the reflection point that is contributing to the signal. In this case, it is the area of the source and the receiver that contribute; depending on the wavelength these areas can also have a radius of more than half a wavelength. This has to be taken into account additionally to the Fresnel zone when considering the spatial resolution.

The problem with the changing waveform is that stacking as an important step in the seismic imaging process assumes that the waveform stays constant, otherwise the superposition of the signals may not add constructively. Hence, we need to determine the critical offset below which stacking improves the signal quality for any applied source frequency.

The waveform as a function of offset can be predicted either numerically, e.g. by finite-element or finite-difference solutions (Savic and Ziolkowski, 1994), or analytically. The solutions depend highly on the geometry of the emitting surface. Therefore, we derive in the following a 3D-semi-analytical solution for the 


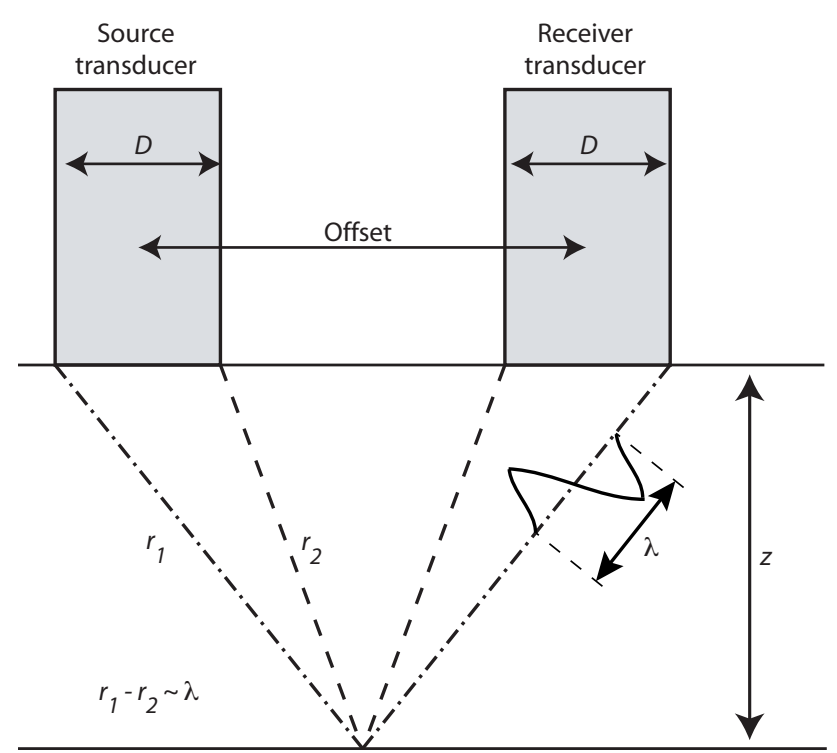

Figure 2.1: Sketch to illustrate that the waveform changes with offset, when high frequency energy propagates between two spatial transducers of diameter $D$. The two sample rays $r_{1}$ and $r_{2}$ show the maximum and the minimum raypath lengths possible for the given source-receiver geometry. The length difference can be greater than a wavelength $\lambda$, so that the shape of the recorded wavelet can differ significantly from the emitted one.

energy-time-distribution as a function of offset and then compare it to experimental data in the results section.

\section{D-semi-analytical solution}

The general idea for this solution is, that a certain amount of source area $A_{s}=\int_{x_{s}} \int_{y_{s}} d x_{s} d y_{s}$ contributes linearly to the energy $E$ arriving at a certain time $t$ at the receiver area $A_{r}=\int_{x_{r}} \int_{y_{r}} d x_{r} d y_{r}$ (Figure 2.2). Assuming an isotropic medium and perfect coupling, the arrival time $t$ is equivalent to the ray path length $l$. Hence, we have to solve the following integral equation:

$E(l)=\int_{x_{s}} \int_{y_{s}} \int_{x_{r}} \int_{y_{r}} \delta\left(x_{s}, y_{s}, x_{r}, y_{r}\right) d x_{s} d y_{s} d x_{r} d y_{r}$,

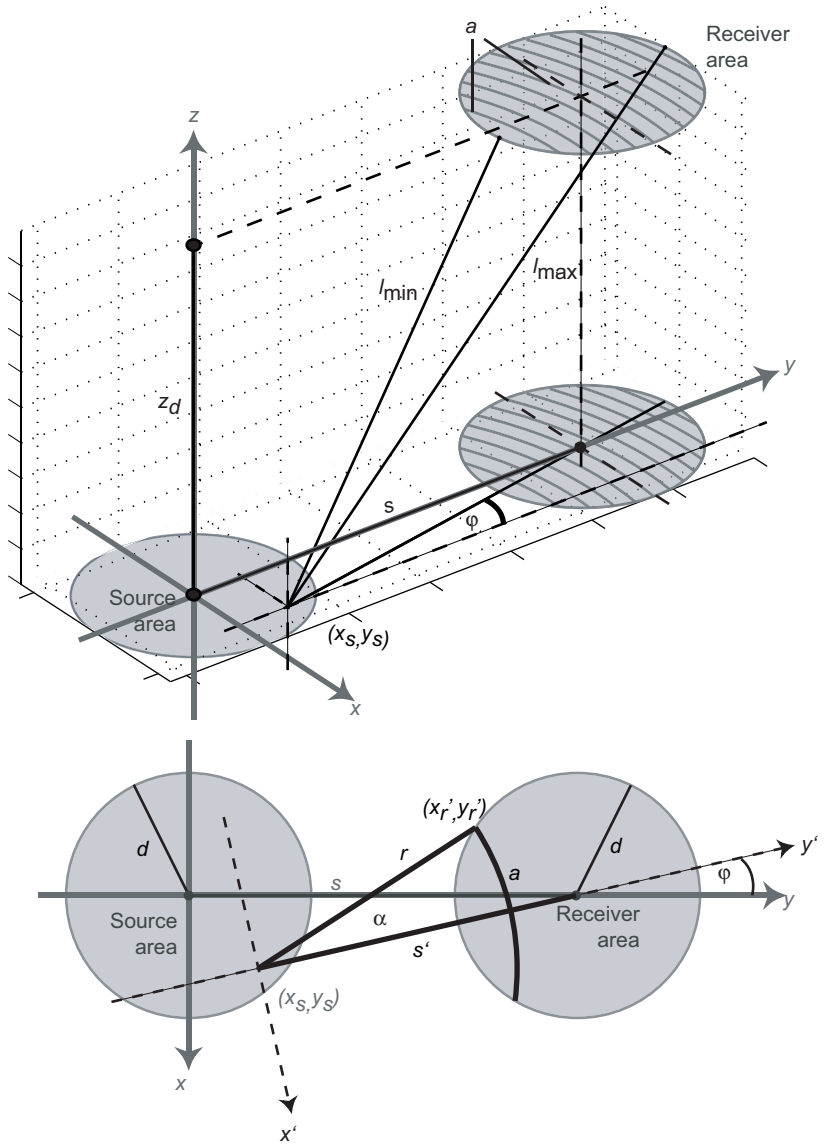

Figure 2.2: Geometrical sketch of all variables needed to calculate the arc length $a$. For detailed explanation, see text and equations 2.1 to 2.11

with

$$
\delta\left(x_{s}, y_{s}, x_{r}, y_{r}\right)=\left\{\begin{array}{rc}
\infty & \text { if }\left(x_{r}-x_{s}\right)^{2}+ \\
& \left(y_{r}-y_{s}\right)^{2}+ \\
& z_{d}^{2}=l^{2} \\
0 & \text { else }
\end{array}\right.
$$

where $z_{d}$ is the vertical distance between the source and the receiver. Each point $\left(x_{s}, y_{s}\right)$ of the source area $A_{s}$ acts as a point source and the ray path length $l$ to any point of the receiver $\left(x_{r}, y_{r}\right)$ can be calculated geometrically. Furthermore, the set of $\left(x_{r}, y_{r}\right)$ that are $l$ apart from $\left(x_{s}, y_{s}\right)$ describe an arc $a$ on the area of the receiver. Hence, for each source point, we calculate the arc length $a$ that contributes to the energy recorded at a certain time $t$, i.e. $l$. In this manner, instead of calcu- 
lating an area, we calculate the arc lengths $a$ for each $l$ and integrate over $A_{s}$ :

$$
A(l)=\int_{A_{s}} a\left(x_{s}, y_{s}, l\right) d A_{s} .
$$

Then, for a given source point $\left(x_{s}, y_{s}\right)$, an offset $s$ to the receiver center and a depth $z_{d}$ between source and receiver, the arc length $a$ can thus be expressed as a function of $l$ :

$$
\begin{aligned}
a_{x_{s}, y_{s}}(l) & =2 r(l) \alpha, \\
r(l)^{2} & =l^{2}-z_{d}^{2}, \\
\alpha(r) & =\tan ^{-1}\left(\frac{x_{r}^{\prime}}{y_{r}^{\prime}}\right), \\
y_{r}^{\prime} & =\frac{1}{2 s^{\prime}}\left(r^{2}-d^{2}+s^{2}\right), \\
x_{r}^{\prime} & =r^{2}-y_{r}^{\prime 2} \\
s^{\prime} & =\left(s-y_{s}\right) \cos (\varphi), \\
\varphi & =\tan ^{-1}\left(\frac{x_{s}}{s-y_{s}}\right),
\end{aligned}
$$

where $d$ is the radius of both source and receiver plane, and $r$ the projection of $l$ onto the $\mathrm{x}$ y-plane. The arc angle $\alpha$ is determined by the intersection $\left(x_{r}^{\prime}, y_{r}^{\prime}\right)$ of the arc and the receiver outline in a new coordinate system $\left(x^{\prime}, y^{\prime}\right)$. This new coordinate system is offset by $\left(x_{s}, y_{s}\right)$ and rotated by $\varphi$ to the old one $(x, y) . s^{\prime}$ is the distance to the center of the receiver plane from the origin of $\left(x^{\prime}, y^{\prime}\right)$ and thus the offset of the receiver to the source point $\left(x_{s}, y_{s}\right)$.

Since the integration of $a$ over $A_{s}$ is not trivial, we evaluate $a(l)$ at regularly spaced, discrete $\left(x_{s}, y_{s}\right)$ positions:

$$
A(l)=\sum_{y_{s}} \sum_{x_{s}\left(y_{s}\right)} a\left(x_{s}, y_{s}, l\right) .
$$

As long as the spacing $d x$ is smaller than the Nyquist theorem requests to prevent spatial aliasing, the discretized solution to $A(l)$ is proportional to the integral solution. Hence, the evaluated waveform and its amplitude are also proportional to a purely analytical solution.

\begin{tabular}{|l|l|}
\hline Source and receiver radius $d:$ & $12 \mathrm{~mm}$ \\
\hline Vertical displacement $z_{d}:$ & $100 \mathrm{~mm}$ \\
\hline Spatial discretization $d x:$ & $0.075 \mathrm{~mm}$ \\
\hline Sampling rate $d t:$ & $50 \mathrm{~ns}$ \\
\hline Offsets $s:$ & every $6 \mathrm{~mm}$ \\
& from 0 to $120 \mathrm{~mm}$ \\
\hline Source frequencies: & $175,250,350,500$, \\
& $750,1000 \mathrm{kHZ}$ \\
\hline
\end{tabular}

Table 2.1: List of parameters used to calculate the waveforms for different offsets and frequencies. The geometry corresponds to that used in the experiments, and the sampling rate is equal to that of the recording equipment in the laboratory.

To obtain the waveform theoretically recorded at offset $s$ and depth $z_{d}, A(l)$ is convolved with the signal emitted by the point source on $A_{s}$, assuming that each point of $A_{S}$ has the same signal. This assumption obviously does not hold true in reality, because the waveform is, during its course from a digital signal to its emission, subject to several interactions that change its shape inherently. To adjust the analytical waveform to resemble the real one, one needs an appropriate weighting function $\omega\left(x_{s}, y_{s}\right)$ to apply to the source area $a\left(x_{s}, y_{s}\right)$. However, we refrain from applying a weighting function or other corrections to the emission function and analyze the changing waveform rather qualitatively. Thus, the energy function is computed for offsets ranging from 0 to $120 \mathrm{~mm}$ and different frequencies using the parameters listed in Table 2.1

The resulting waveforms are shown in Figure 2.3. As it can be clearly seen, the waveforms for smaller offsets (blue) are much more compact and resemble the original waveform, whereas the far offset waveforms (yellow to red) have smaller amplitudes (first column), and later peaks and continue over a longer period of time (center column). The frequency spectra (right column) show that the center frequency of the received signal decreases as the offset and the frequency increases. 

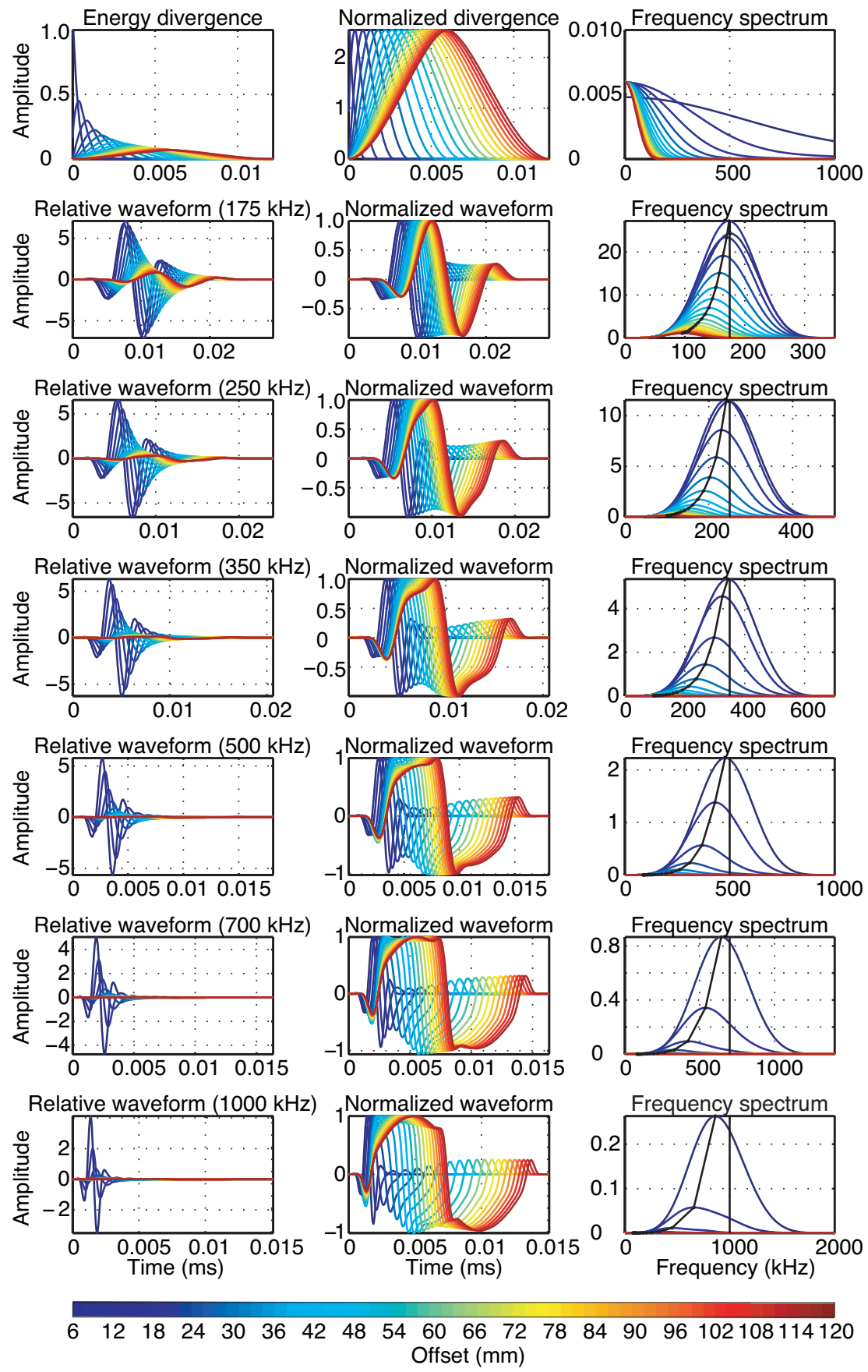

Figure 2.3: Resulting waveforms of the analytical solution for two spatial circular transducers according to the geometry described in Table 2.1 for offsets between 6 (blue) and $120 \mathrm{~mm}$ (red). First row: Temporal energy divergence (relative and normalized) and its frequency spectrum. Second row to last row: Relative and normalized waveforms (source signal convolved with energy divergence function) and the (relative) frequency spectrum for six different source signal frequencies. The vertical black line in the third column delineates the center frequency of the source, the curved line shows the actual frequency maxima being smaller than the source frequency. The waveforms of smaller offsets are much more compact whereas the far offset waveforms have smaller amplitudes and later peaks and continue over a longer period of time. 

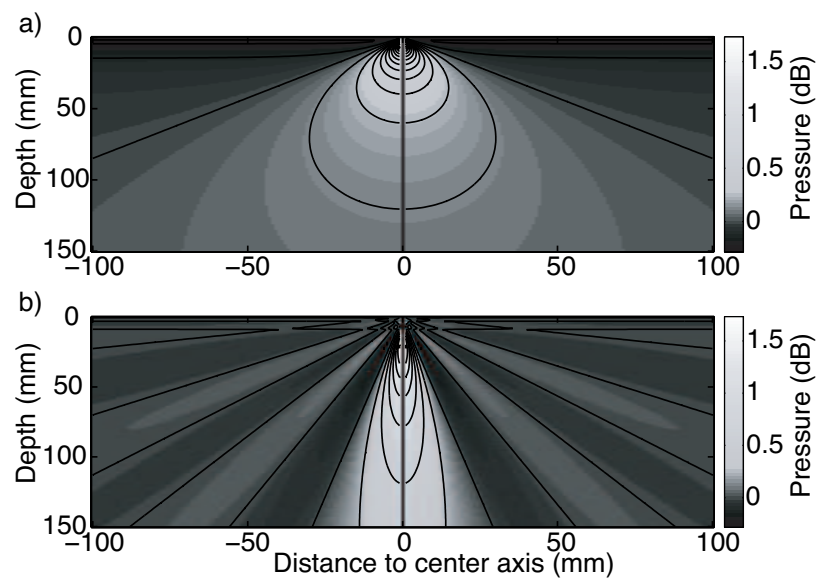

Figure 2.4: The calculated pressure field (see eq.2.12) for a diameter of $12 \mathrm{~mm}$ as a function of depth and angle for $200 \mathrm{kHz}$ (a) and $800 \mathrm{kHz}$ (b). The higher the frequency the more focussed is the beam.

\subsubsection{Directionality}

For circular sources with a flat surface, the spatial divergence of the pressure field can be analytically described as a function of distance to the emitting plane and angle from the axis through the center of the plane by Krautkrämer and Krautkrämer (1986):

$$
p\left(p_{0}, D, \lambda, z, \gamma\right)=4 p_{0} \frac{J_{1}(X)}{X} \sin \left(\frac{\pi D}{8 \lambda z}\right),
$$

with

$$
X=\frac{\pi D}{\lambda} \sin \gamma
$$

where $p_{0}$ is the initial pressure, i.e. amplitude, $D$ the diameter of the emitter, $\lambda$ the wavelength, $z$ the distance to the emitting plane, $\gamma$ the angle to the cylinder axis, and $J_{1}$ the Bessel function. Hence, the higher the applied frequencies and the shorter the wavelengths are, the more directed is the pressure field of a circular transducer as illustrated in Figure 2.4 This implies that less energy propagates at high angles, i.e. far offsets.

However, the emitting plane of the PETs used for this study is made of a piezocrystal of 5-mm

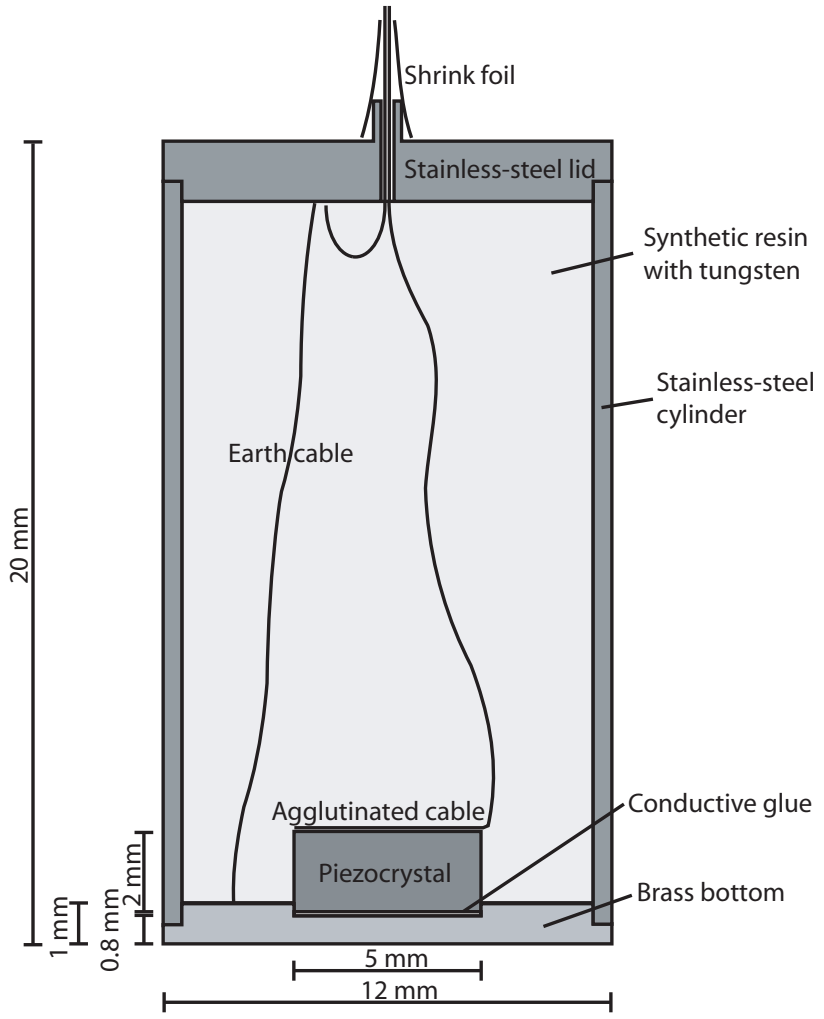

Figure 2.5: Sketch of the cross section of the piezoelectric transducers. The piezocrystal has a diameter of $5 \mathrm{~mm}$ and a height of $2 \mathrm{~mm}$ and is glued onto a brass plate of $12 \mathrm{~mm}$ diameter. For damping, the cylinder is filled with a mix of resin and tungsten. The transducer is in an unexpanded state and the emission area flat.

diameter glued onto a brass plate of $12-\mathrm{mm}$ diameter (Figure 2.5). Hence, both the piezocrystal and the brass plate contribute to the emitted wavefield, such that it has a broader beam (Figure (2.6) at the expense of a smaller amplitude compared to usual PETs of this diameter. Since this is the first time these PETs are used, we determine the effective diameter experimentally by measuring the pressurefield at a fixed distance. We define the effective diameter (Figure 2.6) as the diameter which, when inserted into equation 2.12, gives the best match to the observed amplitudes. This effective diameter is frequency dependent. 


\subsection{Transducer design and ex- perimental setup}

For this study, we used 15 piezoelectric transducers (PET) which were manually build in Germany. The transducers have a 5-mm piezocrystal glued onto a brass plate of 12-mm diameter and 0.8 - $\mathrm{mm}$ thickness underneath the piezocrystal according to Figure 2.5. The brass plate is very thin to keep the effect of internal reflections between the crystal and the plate reasonably small. The resin/tungsten filling is supposed to diminish the resonance ringing of the PET. In order to decrease the effective diameter of our PET, such that its directionality is less pronounced, the emitting plane differs from traditional ones: Commonly, the piezocrystal's diameter is that of the emitting plane (Figure 2.6 left panel) and equation 2.12 applies. In our case, the piezocrystal's diameter of $5 \mathrm{~mm}$ is smaller than the $12-\mathrm{mm}$ diameter of the emitting plane. Hence, both the piezocrystal and the brass plate contribute to the emission such that the effective diameter may be anywhere between 5-12 mm, as illustrated in Figure 2.6 In fact, the bulging emitting surface creates a directivity pattern that is similar to that of a normal transducer of an even smaller diameter, which we define as the effective diameter of our transducers. In the results section, we determine the effective diameter experimentally.

A schematic illustration of the major components of the laboratory seismic system is shown in Figure 2.7 and the specifications are listed in Table 2.2. Briefly summarized, a well defined digital wavelet is converted to an analog electric current and sent to the piezocrystal of the source PET, which in turn expands and contracts according to (the time derivative of) the current. However, despite of the damping material, some ringing remains. Also, interaction between the piezocrystal and the brass plate affects the emitted source wavelet. The effectively emitted source wavelet is therefore different from the digitally inserted signal. After passing through

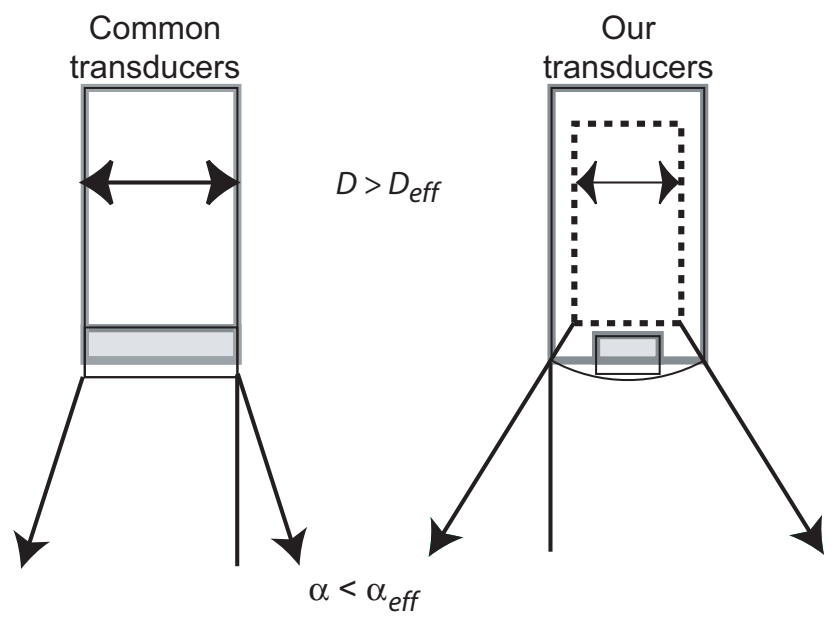

Figure 2.6: Sketch of a traditional piezoelectric transducer versus one of ours with a bulging emitter surface (not to scale). The geometry of an unexpanded piezocrystal is outlined in gray, whereas the expanded geometry is outlined with the thin black line. The traditional transducer has a diameter $D$ and a beam angle $\alpha$, whereas the bulging emitter surface causes the effective beam angle $\alpha_{e f f}$ to be wider and hence the effective diameter $D_{e f f}$ to be smaller (outlined by the dotted black line).

the brass plate, the pulses propagate as pressure waves through room-temperature tap water and are received by a PET of the same build. The process of a digital signal to an analog pressure pulse is reversed for the receiver and the digital signal is recorded and stored in SEG-Y format.

As a source signal we used 2-4 periods of a sine function of the center frequency tapered with a cosine ${ }^{4}$ for six different center frequencies. The shape of the source signal was the same for all applied frequencies. The frequencies ranged between $175 \mathrm{kHz}$ and $1 \mathrm{MHz}$, the sampling rate was $0.05 \mu \mathrm{s}$ and, because the signal showed remarkable consistency, we improved it by vertical stacking 256 times to overcome the weak power output of the transducers.

To determine the directionality as a function of incidence angle and the waveform as a function of offset, two different experiment setups were used as illustrated in Figure 2.8. The geometry for these setups is comparable to that in the sandbox experiments. For these experiments 


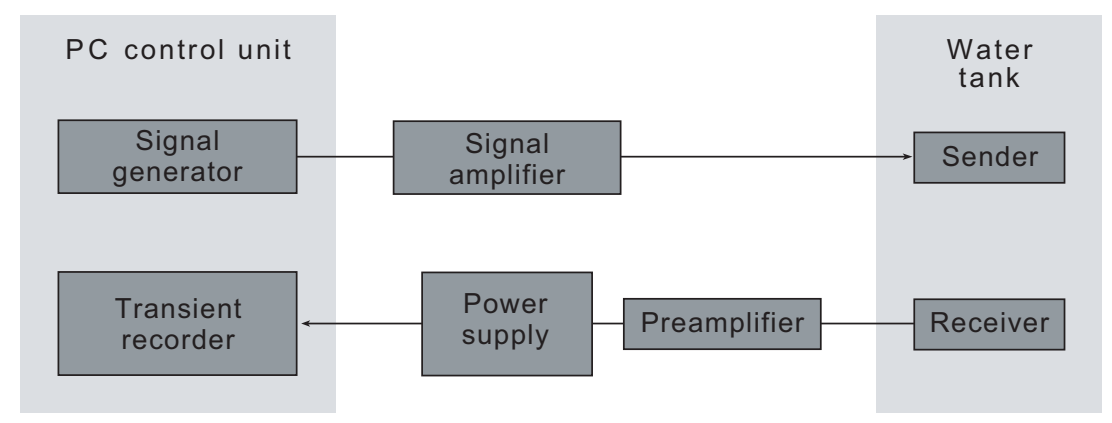

Figure 2.7: Schematic illustration of the functions performed for these experiments by the PC control unit and the positioning system modified after Krawczyk et al. (2007).

\begin{tabular}{|l|l|}
\hline PC system with control unit: & Industry PC (type IPC-9401). \\
\hline Signal generator: & PCI-board (type MI6030); \\
& Max. output $125 \mathrm{MHz}$ (14 bit); \\
& Max. 8 Msamples; \\
& Max. output amplitude $\pm 3 \mathrm{~V}$. \\
\hline Signal amplifier: & AC voltage signal amplifier; \\
& Input -2 to +2 V; \\
& Input resistor $200 \mathrm{Ohm;}$ \\
& Output -141 to $+141 \mathrm{~V} ;$ \\
& Output resistance $2 \mathrm{kOhm;}$ \\
& Band width $20 \mathrm{~Hz}-500 \mathrm{kHz}(-3 \mathrm{~dB}), 20 \mathrm{~Hz}-1000 \mathrm{kHz}(-6 \mathrm{~dB})$. \\
\hline Preamplifier: & (Type VV30) $30 \mathrm{~dB}$ voltage amplification and impedance tuning; \\
& Frequency range $1 \mathrm{kHz}-2 \mathrm{MHz} ;$ \\
& Max. output amplitude $\pm 3 \mathrm{~V}$. \\
\hline Transient recorder: & Three 4-channel PCI-boards (type MI4022); \\
& For each channel signal amplifier and AD-converter; \\
& Max. sampling $20 \mathrm{MHz}(14$ bit); \\
& Max. memory 2 Msamples/channel. \\
\hline
\end{tabular}

Table 2.2: Technical specifications of the components in the ultrasonic recording system (Krawczyk et al., 2007).

the transducers have been tested one by one and at water depths and distances to container walls such that reflections arrive much later in time than the direct waves that are to be analyzed.

The directionality was determined experimentally by opposing two PETs (one source, one receiver) with a constant distance of $10 \mathrm{~cm}$, and rotating the source with a precision of at least $0.25^{\circ}$; the rotation axis was positioned at the emitting end of the source perpendicular to the cylinder axis (Figure 2.8k). The measurements were repeated approximately every $2.15^{\circ}$ from direct incidence up to $43.8^{\circ}$ for six different source frequencies. The maximum amplitudes for each incidence angle and each PET were then automatically picked by a computer.

We define the effective diameter (Figure 2.6) as the diameter that gives the best match between the observed and the theoretical amplitudes from equation 2.12. For this, we implemented a leastsquares inversion routine to find $D, p_{0}$, and $\lambda$ which best match these amplitudes:

$$
\begin{aligned}
& \operatorname{erf}\left(p_{0}, D, \lambda\right)= \\
& \sum_{\gamma}\left[p_{o b s}(\lambda, \gamma)-p\left(p_{0}, D, \lambda, z, \gamma\right)\right]^{2},
\end{aligned}
$$

with

$$
\lambda=c_{w} / f_{s r c},
$$

where the initial pressure $p_{0}$, the diameter $D$, and the wavelength $\lambda$ are the PET and signal parameters, which the error function minimizes for. Additionally, $p_{0}$ is left to be a variable, because the transducers do not respond with equal amplitudes to the electric signal due to their variable sensitivity to frequencies. $\lambda$ is calculated via the wave velocity in water $c_{w}(1500 \mathrm{~m} / \mathrm{s})$ and the center frequency of the source signal $f_{s r c}$. However, because the recorded frequency maxima are smaller 
a) Directivity: Experimental setup

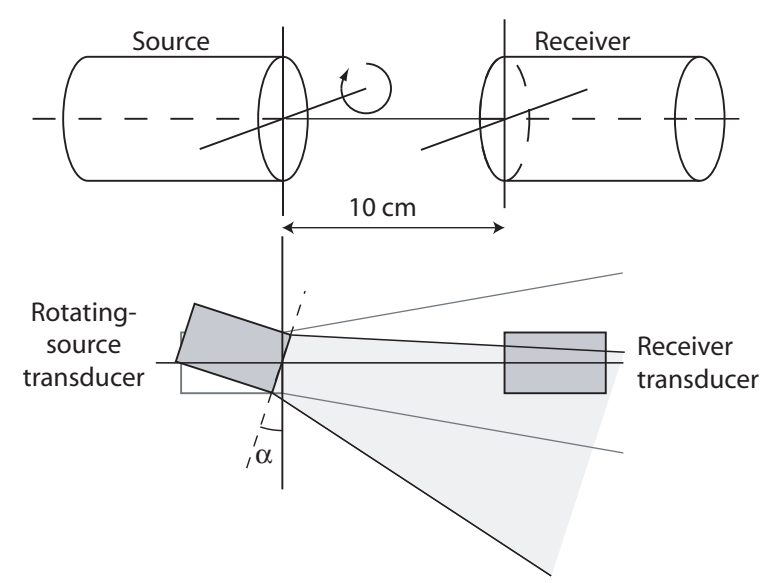

b) Waveform: Experimental setup

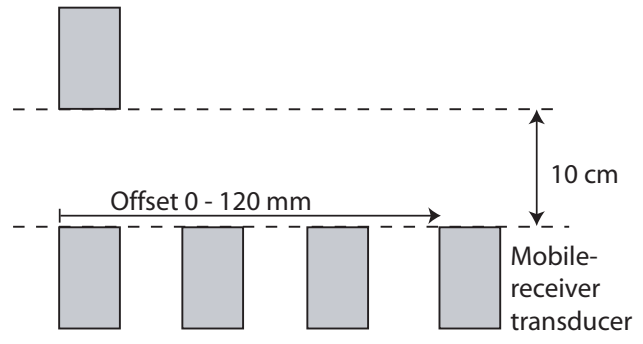

Figure 2.8: Experiment setup for measuring a) the directionality as a function of incidence angle $\alpha$, and b) the waveform as a function of offset.

than the digital center frequencies, we also allow to minimize for $\lambda$ within according boundaries.

The distance between source and receiver $z$ and the angle to the cylinder axis $\gamma$ are determined by the experiment setup. We consistently replace the pressure given in decibel by Krautkrämer and Krautkrämer (1986) by amplitudes in $\mathrm{mV}$ as they are recorded by our system. Hence, in equation 2.15 we minimize for the effective diameter $D$, the initial pressure $p_{0}$, and, within reasonable boundaries, for $\lambda$.

The waveform experimental setup (Figure $2.8 \mathrm{~b}$ ) provides a method for recording the signal as a function of offset by moving the receiving transducer parallel to the emitter surface of the source transducer. The distance $z$ was 10 $\mathrm{cm}$ and the offset varied between 0 and $12 \mathrm{~cm}$. To compare the actual waveforms at different offsets, the arrival time delay due to increasing

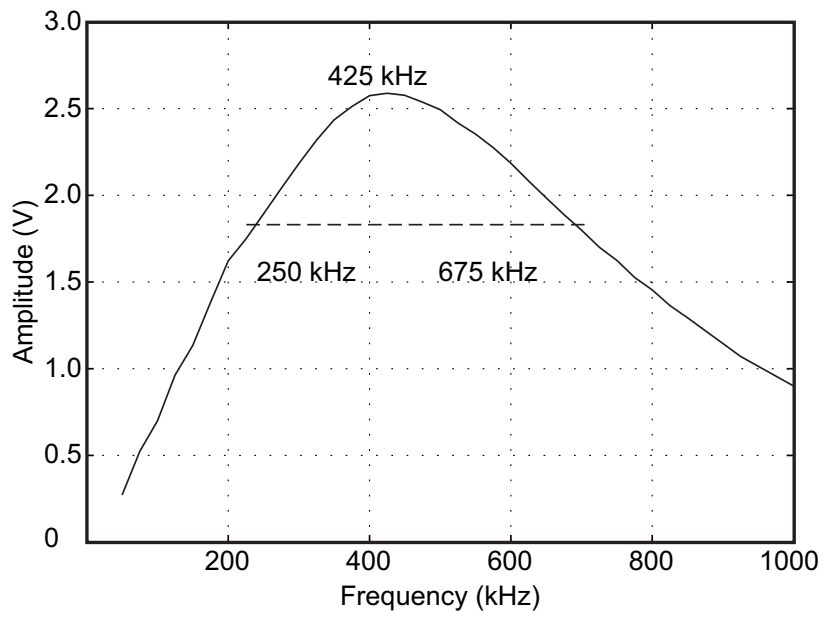

Figure 2.9: Frequency spectrum of piezoelectric transducers used here. The transducers are most sensitive at $425 \pm$ $25 \mathrm{kHz}$ with a half-power bandwidth of 250 to $675 \pm 25$ $\mathrm{kHz}$.

offsets is subtracted accordingly. The receiving transducer was the same for all experiments. The zero-offset traces were used to determine the sensitivity of the PETs to different frequencies and the resonance frequency.

\subsection{Experimental results}

\subsubsection{Resonance}

The measured amplitudes for different source frequencies are displayed in Figure 2.9 The piezoelectric transducers (PET) are most sensitive at $400-450 \mathrm{kHz}$ with a half-power bandwidth of 250 to $675 \mathrm{kHz}$.

\subsubsection{Waveform}

Before analyzing the waveforms, we looked at the length of the source signal, i.e. the number of periods in the source wavelet. We used the waveform experimental setup (Figure 2.8b) and procedure with a source signal of two, three, and four periods of a $350 \mathrm{kHz}$ sine-function tapered with a cosine ${ }^{4}$. Theoretically, for a signal created as described above, the bandwidth is broader, as the 

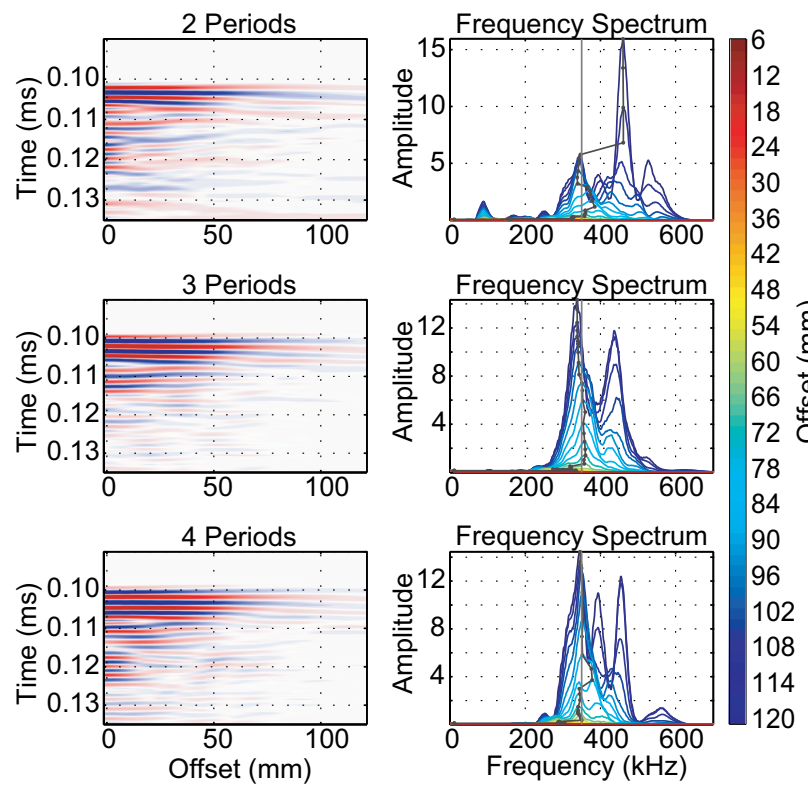

Figure 2.10: Recorded seismograms (350 kHz source frequency) and their frequency spectra for source signals of 2 , 3 , and 4 periods length as a function of offset (color coded). The maximum frequencies are delineated in gray.

signal shortens. However, Figure 2.10 shows that the bandwidth of the recorded signal is equally broad for three different source signal lengths, but the three-period source signal (2nd row panels) produces a better quality spectrum and the second positive phase is clearly defined. The four-period signal requires deconvolution for further use. Hence, for the following experiments, we show the results of the three-period signal experiments.

To analyze the recorded waveforms, we applied different source frequencies using the same experimental setup (Figure 2.8b). In Figure 2.11 we compare the waveform and frequency content for different source frequencies. Aside from the amplitude decay (i.e. directionality), which we deal with in the next section, it is noteable that the first peak arrives later in time for higher offsets, and later phases interfere destructively with varying offsets as is predicted by the analytical solution.

The recorded waveforms look very different to the predicted ones, because the analytical solu- tion does not account for the interactions between the piezocrystal, the damping material and the cylinder walls. The influence of the brass bottom and the glue on the wave propagation are thought to be negligible because their thickness is much smaller than the wavelength. In any case, these interactions can hardly be predicted and may even vary from transducer to transducer. However, the waveforms and amplitudes are comparable in their frequency dependence and therefore the conclusion remains the same:

For all frequencies $<700 \mathrm{kHz}$, the first peak and trough along all offset-traces are in-phase, whereas the end of the signal and the following ringing vary over the offsets. Since we stacked the signal 256 times, we can assume that the ringing is coherent for each offset and use that to our advantage: Since only the first two phases interfere constructively, NMO-stacking reduces the ringing. However, because of the narrow frequency band of the signal, a traditional velocity analysis produces non-unique results. One reflection causes several semblance maxima, one for each peak in the signal at every period of $2-3 \mu \mathrm{s}$, at different velocities. Hence, further processing could be greatly improved by knowing the velocities of the media within a model in advance. Since we know the media that we use to build the model, we can as well measure their velocity beforehand by transmission through a known thickness of the medium.

The strong dependency on the eigenfrequency is revealed by the frequency spectra (Figure 2.11. right column): Each spectrum contains several local frequency maxima; the highest one delineated by the dotted gray line differs from the center frequency of the source (gray line) by \pm 100 $\mathrm{kHz}$ for source frequencies $<1000 \mathrm{kHz}$. The recorded spectra fit the source frequencies when they are between $350-500 \mathrm{kHz}$. The local maxima listed in Table 2.3 show that many maxima are multiples $( \pm 10 \mathrm{kHz})$ of $110 \mathrm{kHz}$. Hence, $110 \mathrm{kHz}$ is the main eigenfrequency. Only the maxima at 165 and $190 \mathrm{kHz}$ cannot be associated 

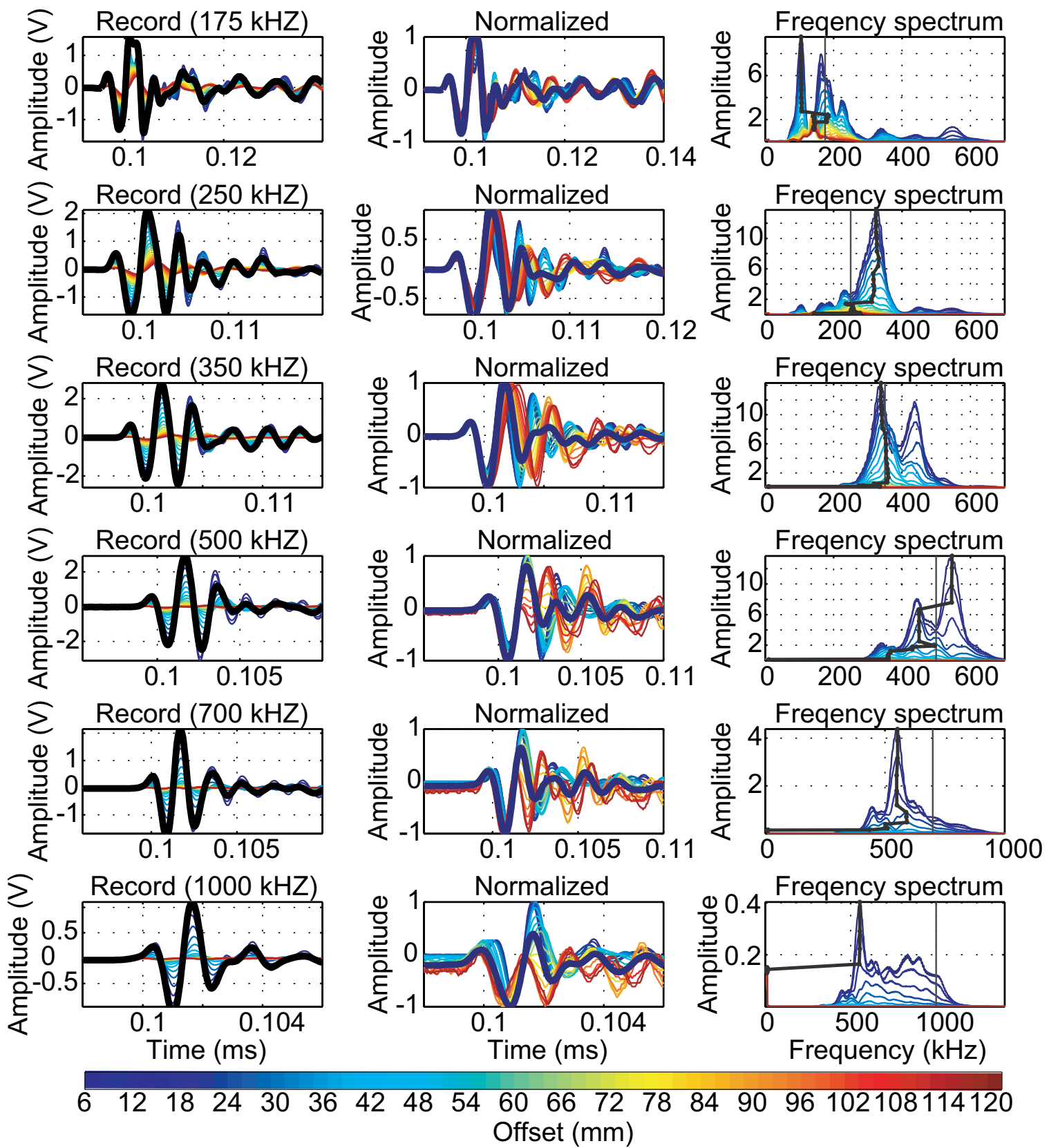

Figure 2.11: Change of recorded waveforms with offset (color code) and frequency (row 1-6) seen in the recorded traces (left), the normalized traces (center), and frequency spectra (right). The stack of all traces is shown in black. The vertical line delineates the source signal frequency, the dotted line the recorded maximum frequencies for all offsets. The first peak and trough are in-phase whereas the end of the signal and the following ringing vary over the offsets. 


\begin{tabular}{|l|l|}
\hline $\begin{array}{l}\text { Source frequency } \\
(\mathrm{kHz})\end{array}$ & Recorded frequency maxima $(\mathrm{kHz})$ \\
\hline 175 & $\mathbf{1 0 5}, \mathbf{1 6 5}, 190,225,340,440,545$ \\
250 & $105,165,190,230, \mathbf{3 2 5}, 450,545$ \\
350 & $\mathbf{3 4 0}, \mathbf{4 4 0}, 540$ \\
500 & $340, \mathbf{4 4 5}, \mathbf{5 5 0}$ \\
700 & $445, \mathbf{5 5 0}$ \\
1000 & $\mathbf{5 5 0}$ \\
\hline
\end{tabular}

Table 2.3: Recorded peak frequencies for different source frequencies. The major maxima are bold.

with the eigenfrequency and can be due to oscillations in a different plane.

Hence, for the preferred source frequencies of $250-675 \mathrm{kHz}$ with respect to the sensitivity, the first two phases are not affected by the changing waveform. Destructive interference for later phases works to our advantage in further data processing. The spectra fit the source frequency best for source frequencies of 350 and $500 \mathrm{kHz}$, and both contain a major peak at $550 \mathrm{kHz}$. This reduces the suitable frequency range to 350-550 $\mathrm{kHz}$, even though all frequencies between 100 to $1000 \mathrm{kHz}$ can be applied to the transducers.

\subsubsection{Directionality}

The seismograms of a 5-mm PET for six different source frequencies, i.e. wavelengths, illustrate how the amplitudes decay with increasing incidence angles (Figure 2.12). This effect of directionality is more pronounced with higher frequencies. At $500 \mathrm{kHz}$ source frequency, the signal cannot be distinguished from ringing noise at an incidence angle $<35^{\circ}$, whereas for $1000 \mathrm{kHz}$, the limiting angle is around $23^{\circ}$. Note, that later phases of the waveform change at around $20^{\circ}$ for frequencies $>350 \mathrm{kHz}$. This is due to the changing geometry with the rotation.

Continuing with this sample PET, we display the maximum amplitudes (Figure 2.13, solid lines) as a function of incidence angle for all tested frequencies (color coded) together with the best-fit curves (dashed lines). These best-fit amplitudes are the result of inserting the parameters of the best-fit solution (eq. 2.15) into the pressure
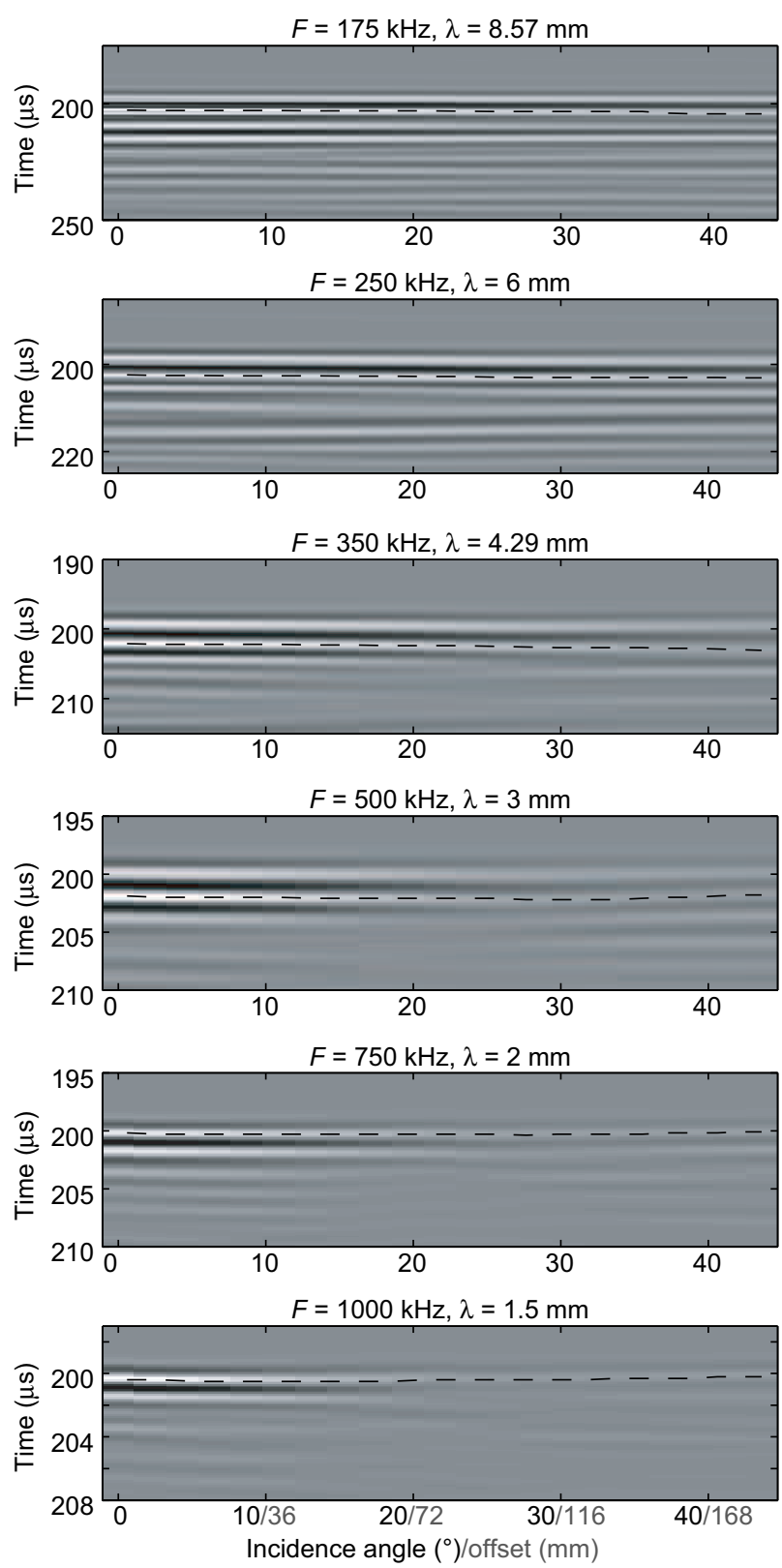

Figure 2.12: Recorded seismograms of a sample transducer as a function of incidence angle for different source frequencies $(F)$, that, in water, are equivalent to the given wavelengths $(\lambda)$. The gain has been maintained at a constant value. The incidence angle increases from left (direct) to right $\left(44^{\circ}\right)$. The respective offset indicated is calculated assuming a reflector depth of $10 \mathrm{~cm}$. The dashed line indicates the picked maximum amplitude used for the minimization. 


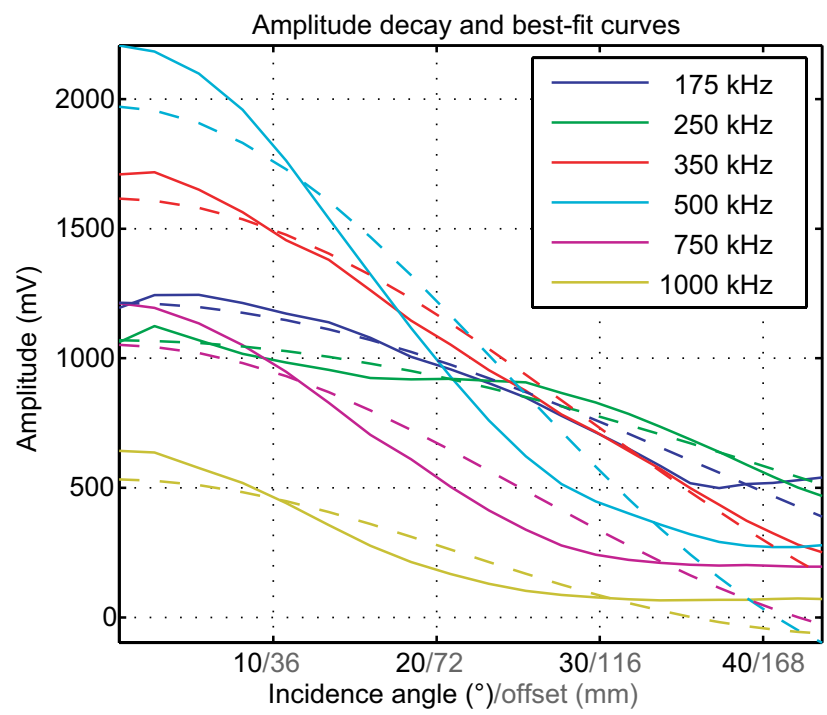

Figure 2.13: Amplitude decay of the sample transducer (solid) and the best-fit theoretical curves (dashed) for different source center frequencies (color coded). The incidence angle increases from left (direct) to right $\left(44^{\circ}\right)$. The respective offset indicated is calculated assuming a reflector depth of $10 \mathrm{~cm}$.

field equation 2.12. The variability of the directincidence values illustrates the sensitivity of the transducers to frequency. The amplitude decay is most prominent for frequencies where the directincidence values are high.

The maximum amplitudes of all PETs are shown in Figure 2.14, Each panel represents one source frequency. The amplitudes of the 5-mm transducers (gray) vary $\pm 500 \mathrm{mV}$ for $350-500$ $\mathrm{kHz}$; however, the amplitude decay follows the same course for all frequencies. The mean decay course is delineated in red and the blue lines represent the best-fit curves of the mean effective parameters. Hence we conclude, that the 5-mm transducers are comparable in their directionality and, for higher frequencies, in their sensitivity. For comparison, the amplitude decay of a tested 7-mm PET (black) has much higher amplitudes at direct incidence and is much more directed. Although the energy output of the 7-mm transducer is better than that of the 5-mm transducers, its strong directivity disinclines us to use it for multiple-offset surveys.
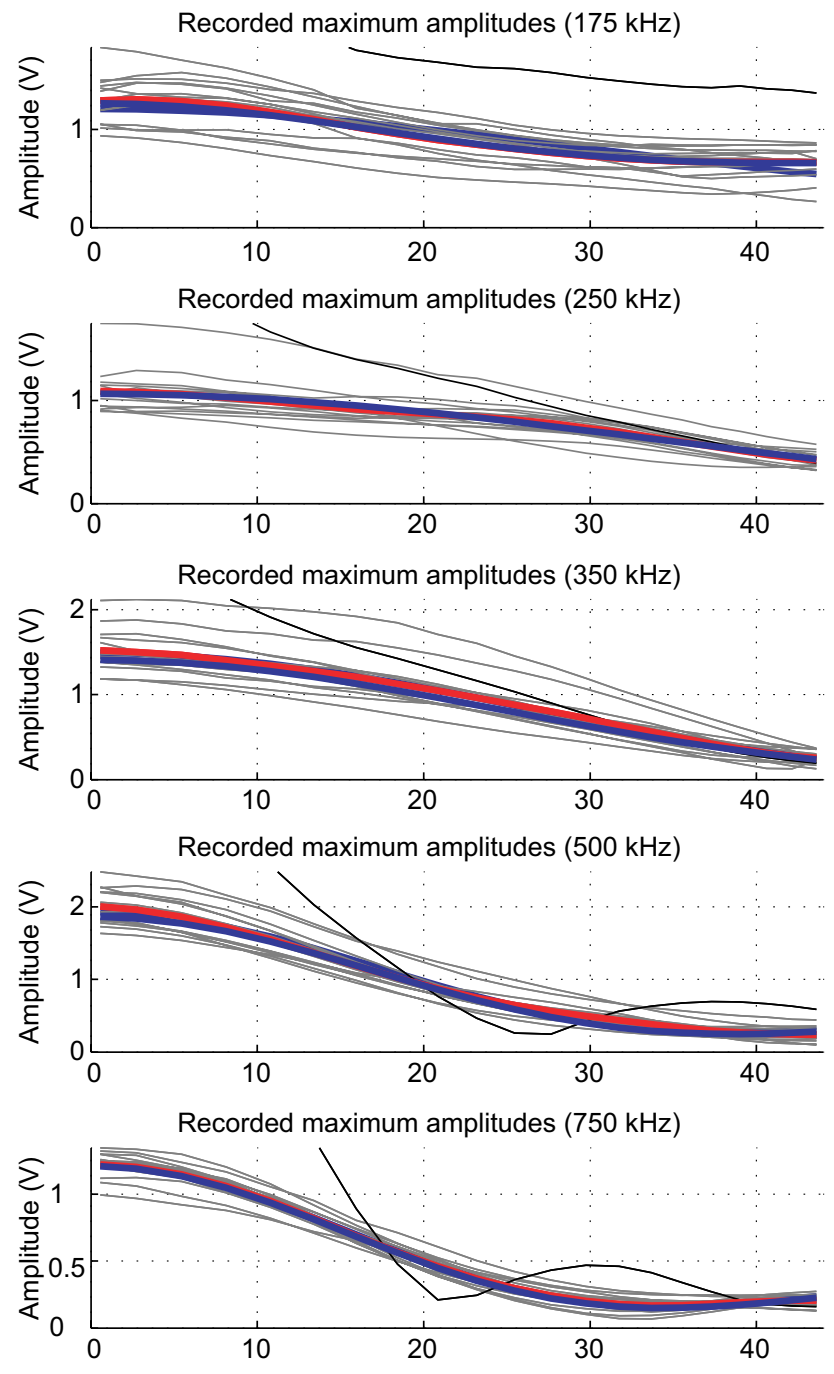

Recorded maximum amplitudes $(1000 \mathrm{kHz})$

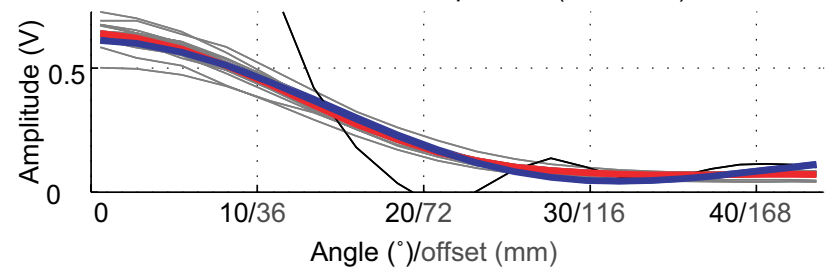

Figure 2.14: Comparison of the amplitude decay of the $5-\mathrm{mm}$ piezoelectric transducers (PET) delineated in gray, their mean (red), their best-fit curve (blue) to the mean results from Figure 2.15 for six different source frequencies. Also shown is the amplitude of a 7-mm PET (black).

The best-fit parameters $\left(D_{e f f}, p_{0}\right.$, and $\left.\lambda\right)$ of all PETs (gray) and their mean (blue) are shown as a function of frequency in Figure 2.15. When these mean values are inserted into equation 2.12 the resulting amplitudes are nearly coincident with 

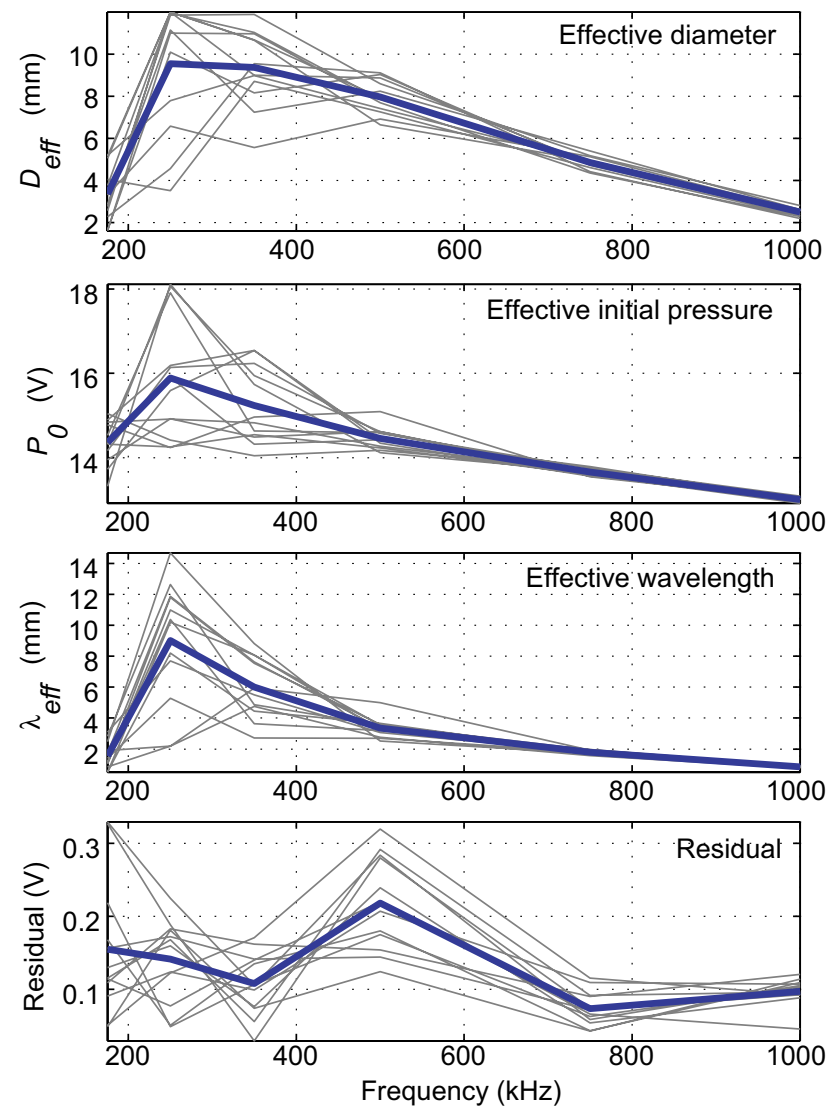

Figure 2.15: Effective parameters (diameter $D_{\text {eff }}$, wavelength $\lambda$, and initial pressure $p_{0}$ ) determined from measurements with different source frequencies. The results for each individual piezoelectric transducer (PET) are displayed in gray and their mean value in blue.

the mean amplitude decay in Figure 2.14 The inversion for the $175 \mathrm{kHz}$ source signal was poorly conditioned and did not converge, therefore the $175 \mathrm{kHz}$ results are not very meaningful and are not discussed in the following. The relatively high standard deviation of the best-fit parameters for the lower frequencies is also a result of the variation in the data. In the high frequency range, the transducers are more similar in their behavior and the inverted parameters are more consistent.

For all transducers, the determined effective diameter is decreasing with increasing frequency (see Table 2.4) and at high frequencies it in fact does go below the piezocrystal's diameter of 5 $\mathrm{mm}\left(\mathrm{D}_{\text {eff }}=4.85 \mathrm{~mm}\right.$ at $\left.\mathrm{f}_{\mathrm{src}}=700 \mathrm{kHz}\right)$. Hence,

\begin{tabular}{|c|c|}
\hline Source frequency $(\mathrm{kHz})$ & $D_{\text {eff }}(\mathrm{mm})$ \\
\hline 175 & $3.36 \pm 1.30$ \\
250 & $9.54 \pm 3.12$ \\
350 & $9.37 \pm 1.81$ \\
500 & $7.96 \pm 0.82$ \\
700 & $4.85 \pm 0.30$ \\
1000 & $2.51 \pm 0.17$ \\
\hline
\end{tabular}

Table 2.4: Mean and standard deviation of effective diameters $D_{\text {eff }}$ for different source frequencies.

our design in fact reduces the effective diameter of the PETs.

\subsection{Discussion}

The experiments tested the piezoelectric transducers (PET) for their frequency sensitivity, and for the change of waveform and directivity as functions of offset and frequency. The results are summarized in Table 2.5

All frequency requirements considered, the best quality results were achieved with a 350-550 $\mathrm{kHz}$ source frequency, because the PETs are most sensitive in that range and the recorded spectra best match the source frequency spectrum. The waveform changes more rapidly the higher the source frequencies are, however, the first two phases of the recorded wavelet are in phase for up to $12 \mathrm{~cm}$ in offset. Later phases interfere destructively to some degree, so that stacking actually focuses the signal. With the experimental setup used here, where the receiver is opposing the source, the effect of the offset on the waveform is not as big as it will be when both source and receivers are on the surface while surveying sandbox models. On the other hand, the velocities in sand and other granular material are around $1700 \mathrm{~m} / \mathrm{s}$ (Sherlock and Evans, 2001), so that the wavelengths increase at the water-model interface. That, and the smaller path difference at deeper offsets counter-balance the waveform change at reflections within the model. Hence, the fit between the first two phases is sufficiently close, so that the changing waveform remains to be a minor problem when both source and receivers are at the surface. During further pro- 


\begin{tabular}{|l|l|l|l|}
\hline PET property & Effect & target & Solution / opportunity \\
\hline \hline Sensitivity & Narrow bandwidth & $250-675 \mathrm{kHz}$ & Apply 250-675 kHz \\
\hline Resonance & Ringy signal & $\mathrm{n} \times 110 \mathrm{kHz}$ & Damping, deconvolution \\
\hline $\begin{array}{l}\text { Spectrum } \\
\text { characteristics }\end{array}$ & $\begin{array}{l}\text { Does the signal match } \\
\text { the source spectrum ? }\end{array}$ & $\begin{array}{l}\text { Conditionally, } \\
\text { yes. }\end{array}$ & $\begin{array}{l}\text { Apply 350-550 kHz, and } \\
\text { apply 3-period signal }\end{array}$ \\
\hline Large dimension & $\begin{array}{l}\text { Changing waveform } \\
\text { (temporal } \\
\text { divergence) }\end{array}$ & $\leq 700 \mathrm{kHz}$, & $\begin{array}{l}\text { - Positive interference } \\
\text { for first peak and trough } \\
\text { - Negative interference } \\
\text { for later phases } \\
\end{array}$ \\
& & $\begin{array}{l}\text { - A-priori knowledge of } \\
\text { material velocity recommended }\end{array}$ \\
\hline Directivity & $\begin{array}{l}\text { Less energy } \\
\text { at high offsets }\end{array}$ & $<35^{\circ}$ & $\begin{array}{l}\text { - Increase water depth } \\
\text { - Reduce } D_{\text {eff }}\end{array}$ \\
\hline
\end{tabular}

Table 2.5: List of the piezoelectric transducer (PET) properties, the resulting target frequencies, incidence angles, and offsets, and other solutions or alternatives.

cessing steps, the image quality could be greatly improved by knowing the velocities of the media within a model in advance. Since we know the media that we use to build the model, we can as well measure their velocity beforehand by sounding through a known thickness of the medium.

An alternative to this approach is to use the apriori knowledge of the waveform to our advantage: Because the waveform is a function of offset and reflector depth, the energy distribution is unique for each offset, and we can use it to determine the reflector depth. Additionally, if the specific waveform for each offset is known, a shaping filter or cross-correlation wavelet can be designed for each offset, so that after filtering, the signals are sufficiently similar for a stack. Only we need to know the waveform for each offset and depth. The disadvantage of this solution is that the amplitude decays more than the waveform changes with increasing offset. Hence, it is more applicable to stay within the offset limits imposed by the directionality. The analytical solution to the changing waveform problem predicts the temporal divergence of the signal and shows that the loss of high frequencies is due to different path lengths and not to attenuation alone. The shape of the signal though is very different to the recorded shapes because the analytical function does not respect the interactions between the piezocrystal, the damping material, and the cylinder walls. Hence, the analytical solution in no way can replace experiments.
For all our experiments, we chose a water depth of $10 \mathrm{~cm}$ comparable to the water depth in experiments where sandbox models are seismically surveyed. At this water depth level, a $20-\mathrm{cm}$ offset is equivalent to an incidence angle of $45^{\circ}$. The directivity results though suggest, that the incidence angle should not fall below $35^{\circ}$ for a source frequency of $500 \mathrm{kHz}$, which corresponds to an offset of $14 \mathrm{~cm}$. To increase applicable offsets, the water depth has to be increased. Furthermore, an increased water depth reduces the temporal divergence due to smaller length differences of raypaths.

In conclusion, we are restricted to 350-550 $\mathrm{kHz}$ and water depths $\geq 10 \mathrm{~cm}$. Hence, the emitted wavelength is between $4.2-2.7 \mathrm{~mm}$, allowing to resolve features of $\sim 2-1.5 \mathrm{~mm}$ vertical dimension. This frequency range is very suitable to image the structures in sandbox models. Higher frequencies generate wavelengths that approximately as big as the grain size of the material, so that each grain causes a reflection and obscures the image of larger structures, whereas smaller frequencies do not resolve the structures in sandbox models. The lateral resolution is dependent on the p-wave velocity of the medium, the depth of the reflection, and in this case of the effective diameter of the PETs, which has to be added as a constant to the normal fresnel zone calculation. In the worst case, i.e. the lowest source frequency of $350 \mathrm{kHz}$ source signal and the deepest reflection at $15 \mathrm{~cm}$ through wet sand, it is going to be 
about $46 \mathrm{~mm}$. The models, therefore, should contain lateral variation longer than that, and shear bands should cut through at least a 46-mm width.

To do amplitude-versus-offset (AVO) analyses on field data, very often a plane-wave solution (Zoeppritz) is used, although the wave propagation is spherical. Alhussain et al. (2008) have experimentally confirmed the spherical wave effect on the AVO response by laboratory ultrasonic measurements using omni-directional pwave transducers with a $220 \mathrm{kHz}$ dominant frequency. The plane-wave solution (Zoeppritz) that is widely used for AVO analysis agrees well for moderate incidence angles up to $25^{\circ}$ (their Figure 2), but is not valid at large angles. However, within the beam our PETs produce something between a plane wave and a spherical wave, and hence the AVO implementations are applicable to data collected with our PETs approximately up to incidence angles of $32^{\circ}$.

Further improvement of the sources and receivers can only be achieved by using smaller transducers that have the same or higher energy output and the same frequency bandwidth. Unfortunately, those transducers cannot be fabricated yet. However, our design of a smaller piezocrystal being glued onto the brass plate did reduce the effective diameter, at high frequencies even to diameters less than that of the piezocrystal. Further improvement of this technique possibly will lead to transducers with zero effective diameter and high energy output.

The next step on the way towards seismic imaging of sandbox models, is to find suitable granular material. In the experiments reported here, the transmitting medium was water. The energy losses at the grain-to-grain contacts are very high, so that the penetration depth is not expected to be more than a few centimeters. Careful sieving and saturation with boiling or hot water can reduce attenuation caused by unwanted scattering or attenuation due to remaining air bubbles. In a two layer model, energy arriving at the first material interface should be reflected and re- fracted to approximately equal amounts, so that a sufficient amount of energy passes into the second medium to resolve structures within. To create such models, we can measure the velocity and density of available materials to find suitable impedance contrasts, and at the same time we can bypass a velocity analysis in the processing.

\subsection{Conclusion}

In this study, we tested the properties of specially designed piezoelectric transducers (PET) for their usefulness in seismic reflection surveys of sandbox models, covering three aspects, namely the frequency sensitivity, the directionality, and the changing waveform. We presented an analytical solution to the changing waveform and compared it to the recorded ones.

The special design of our PETs, where a smaller diameter $(5 \mathrm{~mm})$ piezocrystal is glued onto a bigger diameter $(12 \mathrm{~mm})$ brass plate, in fact reduced the directionality, so that the effective diameter is smaller than that of traditional PETs. The directivity experiments show, that the incidence angle should not fall below $35^{\circ}$ for a source frequency of $350-550 \mathrm{kHz}$, which corresponds to an offset of $14 \mathrm{~cm}$ for a water depth of $10 \mathrm{~cm}$. The changing waveform is a minor problem for those offsets and frequencies. However, an a-priori knowledge of the p-wave velocity of the materials is recommended to bypass the velocity analysis during further processing. The analytical solution to the changing waveform problem predicted the temporal divergence of the signal and showed that the loss of high frequencies is due to different path lengths and not to attenuation alone. However, it did not reproduce the recorded waveforms because it does not respect the internal dynamics of the PETs.

With respect to the PETs, we have shown that reflection processing on such a small scale is feasible for source frequencies of $350-550 \mathrm{kHz}$ and for incidence angles $<35^{\circ}$. This frequency range 
allows to resolve structures of $\sim 2-1.5 \mathrm{~mm}$ dimension, which is sufficient to resolve the structures within sandbox models. However, the bandwidth of the generated signals is relatively small for seismic surveying. In order to broaden the spectrum, we recommend to pulse in several of these narrow frequency bands and stack those as a preprocessing step. 